\title{
Quality of life and cost-effectiveness of interferon-alpha in malignant melanoma: results from randomised trial
}

\author{
S Dixon ${ }^{*, 1}$, SJ Walters', L Turner ${ }^{2}$ and BW Hancock ${ }^{2}$ \\ 'Health Economics and Decision Science, and Trent Research and Development Support Unit, School of Health and Related Research, University of \\ Sheffield, Regent Court, 30 Regent Street, Sheffield SI 4DA, UK; ${ }^{2}$ Academic Unit of Clinical Oncology, University of Sheffield, Weston Park Hospital, \\ Whitham Road, Sheffield SIO 2SJ, UK
}

\begin{abstract}
A definitive conclusion regarding the value of low-dose extended duration adjuvant interferon-alpha therapy in the treatment of malignant melanoma is only possible once data on health-related quality of life (HRQoL) and costs have been considered. This trial randomised 674 patients to interferon alpha-2a (3 megaunits three times per week for 2 years or until recurrence) or placebo. Health-related quality of life (QoL) was to be assessed up to 60 months using the European Organisation for Research and Treatment of Cancer (EORTC) QLQ-C30. Data for the economic analysis, including cost information and the EQ-5D were also collected. Patients in the observation (OBS) group had significantly better mean follow-up quality of on five dimensions of the EORTC QLQ-C30 functional scales: role functioning $(P=0.033)$, emotional functioning $(P=0.003)$, cognitive functioning $(P=0.00 \mathrm{I})$, social functioning $(P=0.003)$ and global health status $(P=0.00 \mathrm{I})$. Patients in the OBS group had significantly better mean follow-up symptom scores on seven dimensions of the EORTC QLQ-C30 VI symptom scales. Economic data showed that costs were $£ 3066$ higher in the interferon group and produces an incremental cost per quality-adjusted life year of $£ 4$ I 432 at 5 years. The results show that interferon has significant effects on QoL and symptomatology and is unlikely to be cost-effective in this patient group in the UK.

British Journal of Cancer (2006) 94, 492-498. doi:I0.1038/sj.bjc.6602973 www.bjcancer.com
\end{abstract}

Published online 3I January 2006

(C) 2006 Cancer Research UK

Keywords: interferon-alpha; adjuvant; melanoma; quality of life; cost-effectiveness

The role of interferon-alpha in malignant melanoma has long been the debated and researched, with over 6000 patients entered into trials (Molife and Hancock, 2002). One aspect of this has been the effectiveness of low-dose extended duration adjuvant therapy. A recent study in 674 patients with thick primary cutaneous melanoma showed no significant difference in overall survival or recurrence-free survival up to 5 years (Hancock et al, 2004). This, together with other trial evidence (Ascierto et al, 2006), points to there being no routine role for low-dose therapy within this patient group.

A definitive conclusion is not possible, however, until data on health-related quality of life (HRQoL) and costs have been considered alongside the survival data. It is possible that a HRQoL advantage exists, or that the cost differentials are such that the treatment may be considered cost-effective even in the face of nonsignificant clinical findings.

Data from within AIM-High have been reported on toxicity and change in Karnofsky Performance Status (Hancock et al, 2004), but these data offer only a partial view of HRQoL. This paper reports on the HRQoL data from AIM-High plus cost and costeffectiveness as estimated by an incremental cost per qualityadjusted life year (QALY).

*Correspondence: S Dixon, E-mail: s.dixon@shef.ac.uk Received 4 November 2005; revised 4 January 2006; accepted 5 January 2006; published online 31 January 2006

\section{MATERIALS AND METHODS}

Patients within the study were randomised to either interferon alpha-2a at 3 megaunits three times per week for 2 years or until recurrence, or placebo. The study protocol was approved by the relevant research ethics committee and all participating patients gave informed written consent.

HRQoL data in the form of the European Organisation for Research and Treatment of Cancer (EORTC) QLQ-C30 were originally intended to be collected at baseline, 3, 6, 12, 24, 36, 48 and 60 months for a subgroup of patients. However, HRQoL data were actually collected at a variety of time points postrandomisation from 3 days to 77 months. Data for the economic analysis, including cost information and the EQ-5D were collected at 3,6 , $12,24,36,48$ and 60 months. These economic data were only collected for a subgroup of patients, selected as every fifth patient to enter the study.

The European Organisation for Research and Treatment of Cancer (EORTC) QLQ-C30 is a 30-item cancer-specific instrument designed to assess the health-related quality of life (QoL) of cancer patients participating in international clinical trials (Aaronson et al, 1993). The QLQ-C30 version 1.0 used in the AIM-High Trial incorporated five functional scales: physical $(\mathrm{PF})$, role (RF), cognitive (CF), emotional (EF) and social (SF); three symptom scales: fatigue (FA), pain (PA), nausea and vomiting (NV); a global health status/QoL scale (QL) and six single items assessing additional symptoms commonly reported by cancer patients: 
dyspnoea (DY), loss of appetite (AP), insomnia (SL), constipation (CO), diarrhoea (DI) and a single item on the perceived financial impact of the disease (FI). All of the scales and single-item measures range in score from 0 to 100. A high scale score represents a higher response level. Thus a high score for a functional scale represents a high/healthy level of functioning; a high score for the global health status/QoL represents a high QoL; but a high score for a symptom scale/item represents a high level of symptomatology/problems.

Patient utilities were obtained from the EQ-5D questionnaire. The EQ-5D is a five-dimensional health state classification. The five dimensions are mobility, self-care, usual activities, pain/ discomfort and anxiety/depression. These five dimensions are each assessed by a single question on a three point ordinal scale (no problems, some problems, extreme problems). An EQ-5D 'health state' is defined by selecting one level from each dimension. A total of 243 health states are thus defined. Values or preference weights for a sample of these health states were obtained from a general community sample using a Time-Trade-Off (TTO) technique. Estimates for all health states were extrapolated from this sample by statistical regression modelling. The EQ-5D preference-based measure can be regarded as a continuous outcome scored on a -0.59 to 1.00 scale, with 1.00 indicating 'full health' and 0 representing dead. The negative EQ-5D scores represent certain health states valued as worse than dead.

Data on resource use covered all key areas of care; interferon dose, inpatient and outpatient hospital care, community nurse and general practitioner care. Data on interferon were collected via the study case report form as completed by the study clinician or research nurse. The other economic data, including the EQ-5D, were collected through a patient completed questionnaire.

\section{Analysis}

Data for the EORTC QLQ-C30 were scored using the EORTC Scoring Manual (Fayers et al, 1995). EQ-5D data were scored using
UK population values (Dolan, 1997), and combined with mortality data to calculated QALYs (Drummond et al, 1997). As baseline EQ$5 \mathrm{D}$ values were missing for baseline, these were imputed from a regression of EORTC QLQ-C30 responses on EQ-5D values from other visits.

Differences in patient characteristics between groups were tested for using independent sample $t$-tests, or $\chi^{2}$ tests, as appropriate. The Kaplan-Meier method was used to calculate the time from randomisation to death, and the log rank test to compare the survival times of both groups (Altman, 1991). HRQoL data were collected at a variety of time points postrandomisation from 3 days to 2136 days, mean 403 days. Patients had between 1 and 13 followup QoL assessments, with an average of 3.85 assessments postrandomisation. Given this variation in data collection we decided on a relatively straightforward approach to the analysis of the longitudinal data which involved the use of summary measures (Matthews et al, 1990). We summarised follow-up QoL responses for each individual subject by taking the simple average of their follow-up QoL responses over time as our summary measure (Matthews et al, 1990) as we were concerned with differences in overall levels of QoL rather than more subtle effects.

Differences in mean follow-up HRQoL between the groups were compared using a multiple linear regression model, with mean follow-up HRQoL as the outcome variable and baseline HRQoL, overall survival status (dead or censored) and treatment group as covariates. $P$-values of less than 0.05 were regarded as being statistically significant.

The economic analysis followed guidelines set down by the National Institute for Clinical Excellence (2004). Costs were calculated by combining resource use data with unit costs representing national estimates (British Medical Association, 2002; Netten and Curtis, 2003). Costs beyond 1 year were discounted at $3.5 \%$ per annum. Prices are at 2003/4 levels with prices adjusted using the Hosptial and Community Health Services Pay and Price Index where appropriate (Netten and Curtis, 2003). Cost differences were tested for using independent sample $t$-tests.

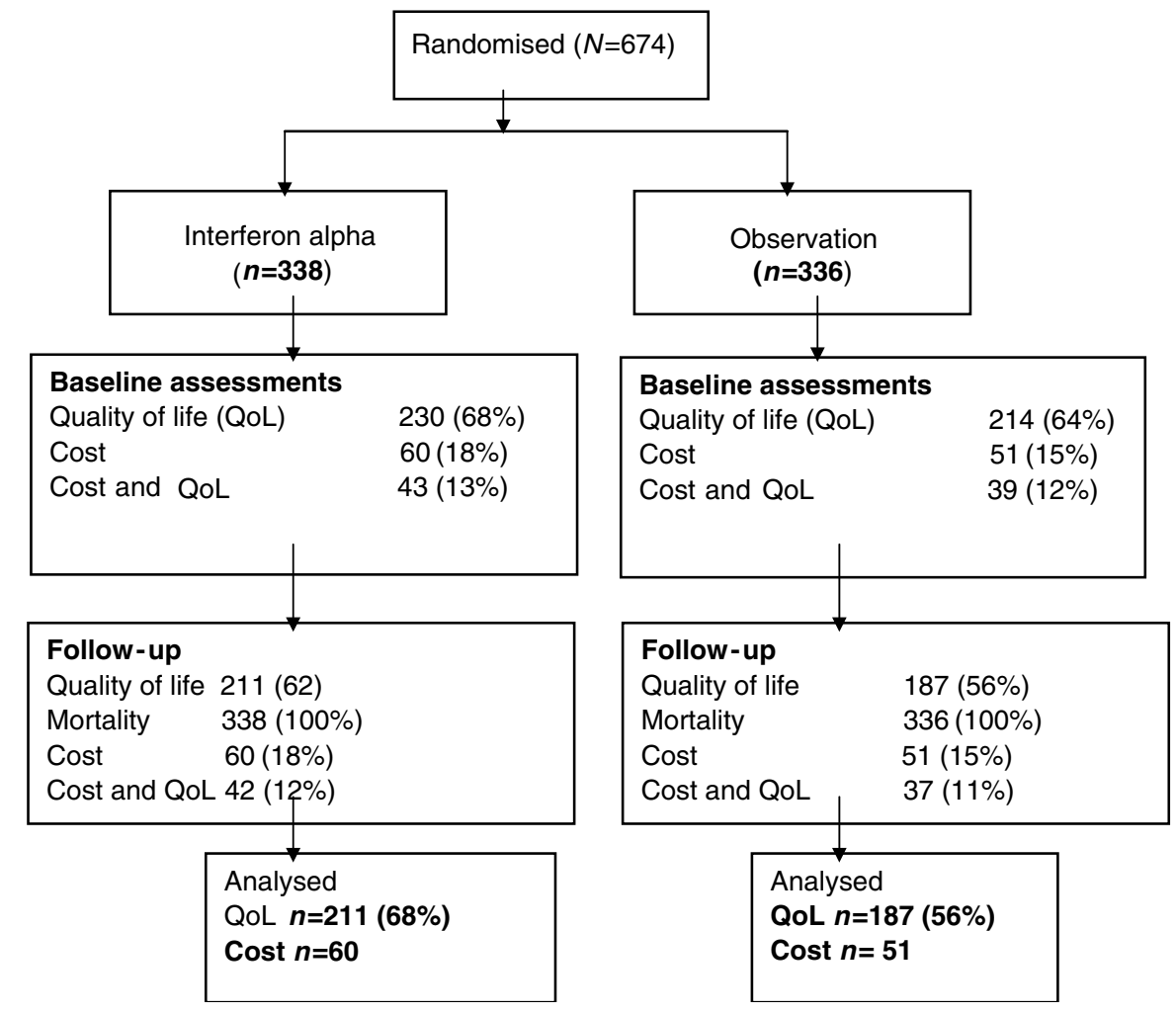

Figure I Enrolment, treatment and follow-up of study patients. 
An incremental cost-effectiveness ratio was calculated using mean costs and QALYs.

Economic data can be severely limited by missing data as both the costs and the QALYs are cumulative measures (i.e. totals over the entire follow-up period). Consequently, if only one value is missing from the full series of follow-up data, the total cannot be calculated. To avoid this problem, missing data imputation becomes an important part of analysing economic data. Within this study, the last observation carried forward was used to impute missing data in order to calculate total costs and QALYs (Heyting et al, 1992).

\section{RESULTS}

\section{Health-related QoL}

Figure 1 shows that 444 patients (out of 674 ) or $66 \%$ had a valid baseline QoL assessment; 230/338 (68\%) in the IFN group

Table I Comparison of samples analysed

\begin{tabular}{|c|c|c|c|c|c|c|c|c|}
\hline & \multicolumn{2}{|c|}{ Economic sample $(n=I I I)$} & \multicolumn{2}{|c|}{ HRQoL sample $(n=444)$} & \multicolumn{2}{|c|}{ HRQoL follow-up sample $(n=398)$} & \multicolumn{2}{|c|}{ Full sample $(n=674)$} \\
\hline & $n$ & $\%$ & $n$ & $\%$ & $n$ & $\%$ & $n$ & $\%$ \\
\hline Censored & 58 & 52.3 & 202 & 45.5 & 186 & 46.7 & 323 & 47.9 \\
\hline Died & 53 & 47.7 & 242 & 54.5 & 212 & 53.3 & 351 & 52.1 \\
\hline Total & 111 & 100.0 & 444 & 100.0 & 398 & 100.0 & 674 & 100.0 \\
\hline \multicolumn{9}{|l|}{ Gender } \\
\hline Male & 69 & 62.2 & 258 & 58.1 & 232 & 58.3 & 382 & 56.7 \\
\hline Total & 111 & 100.0 & 444 & 100.0 & 398 & 100.0 & 674 & 100.0 \\
\hline \multicolumn{9}{|l|}{ Stage } \\
\hline L & 6 & 5.4 & 41 & 9.2 & 35 & 8.8 & 74 & 11.0 \\
\hline LM & 23 & 20.7 & 89 & 20.0 & 77 & 19.3 & 130 & 19.3 \\
\hline RMD & 16 & 14.4 & 53 & 11.9 & 46 & 11.6 & 85 & 12.6 \\
\hline Mean & 51.59 & & & 51.6 & 51.4 & & & 52.0 \\
\hline (s.d.) & 13.29 & & & 12.9 & 12.7 & & & |3.1 \\
\hline
\end{tabular}

$\mathrm{L}=$ localised; $\mathrm{LM}=$ locally metastatic; $\mathrm{RMD}=$ regionally metastatic at diagnosis; $\mathrm{RMR}=$ regionally metastatic at recurrence.

Table 2 Baseline clinical characteristics and QoL in control and intervention $(n=398)$

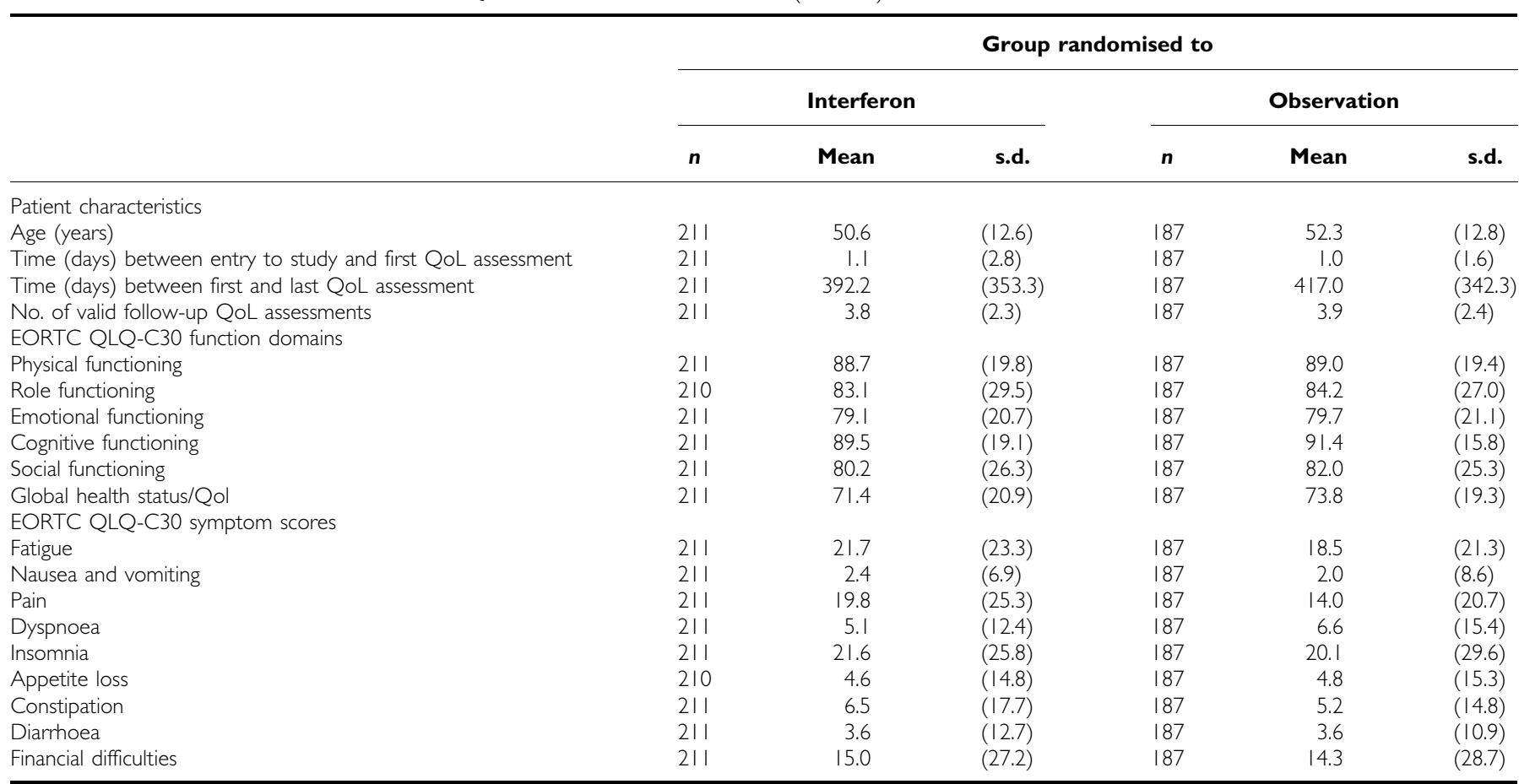

For the EORTC QLQ-C30 vI function scales a higher score represents a better level of functioning. For the EORTC QLQ-C30 vI symptom scales a higher score represents a worse level of symptoms. 
and $214 / 336(64 \%)$ in the OBS group $(P=0.233)$. Comparison of the $n=398$ patients with a valid baseline QoL assessment and at least one valid follow-up QoL assessment and $n=276$ patients with no baseline or follow-up QoL assessments, suggested that the two groups have similar age $(P=0.151)$, gender $(P=0.349)$, histology $(P=0.078)$, and lengths of follow-up $(P=0.528)$ (Table 1$)$.

There was no interaction between treatment group and followup QoL assessment status with regard to overall survival $(P=0.251)$ and no evidence of a difference in overall survival between the no follow-up QoL data and valid follow-up data groups (log rank $P=0.84$ ). Median survival was 4.05 years for patients with no valid follow-up QoL data $v s 3.81$ years for patients with valid baseline and follow-up QoL data. This implies we can assume that the QoL sample of 388 patients is a randomly selected subsample of the AIM-High trial population.

The IFN and OBS groups in the QoL sample had similar age, gender, stage and overall mortality. The IFN and OBS groups in the QoL sample had similar baseline EORTC QLQ-C30 scores, except for the PAIN dimension, where the IFN had significantly higher levels of pain, +5.8 ( $95 \%$ CI: +1.2 to $+10.4, P=0.013$ ), see Table 2. There was no evidence of a difference in overall survival between the IFN and OBS groups $(\log$ rank $P=0.15)$ in the QoL sample. Median survival for IFN was 4.29 years $v s 3.21$ years for the OBS group (see Figure 2).

Patients in the observation (OBS) group had significantly better mean follow-up QoL on five dimensions of the EORTC QLQ-C30 V1 functional scales: RF, EF, CF, SF and QL (see Table 3) after adjustment for baseline QoL and overall survival status (dead or censored). Patients in the OBS group had significantly lower (better) mean follow-up QoL symptom scores on seven dimensions of the EORTC QLQ-C30 V1 symptom scales: FA, NV, DY, AP, CO, DI and FI (see Table 4) after adjustment for baseline QoL and overall survival status (dead or censored).

\section{Economic evaluation}

In total, 134 patients were entered into the economic study and data were available for 111 of these patients. Costs were higher for the interferon (IFN) group in the first 2 years, then slightly lower, thereafter. Overall, costs were $£ 3066$ higher in the IFN group. This is almost entirely due to the cost of therapy (Figure 3), but is not statistically significant $(P=0.396)$. The IFN group generates 0.074 more QALYs (Table 5), which is equivalent to an extra 27 days in full health, although this is not statistically significant $(P=0.752)$.

The incremental cost per QALY for interferon therapy is $£ 41432$. There is considerable statistical uncertainty around this estimate, and a threshold of $£ 30000$ per QALY, there is only a $45 \%$ chance of interferon being cost-effective.

\section{DISCUSSION}

These results show that HRQoL is worse in the IFN group in terms of both functioning and symptomatology. As assessed by the EORTC QLQ-C30, statistically significant differences were found in terms of role functioning, emotional functioning, cognitive functioning, social functioning and global health status. Symptom scores in the IFN group were significantly worse for fatigue, nausea/vomiting, dyspnoea, appetite loss, constipation and diarrhoea.

Despite the great interest in interferon therapy for melanoma and its recognised toxicities (Hancock et al, 2000), there are very few large scale studies that have used validated HRQoL instruments. Paterson looked at 21 patients receiving high-dose interferon alpha-2b using the Functional Assessment of Cancer Therapy - Biological Response Modifier (FACT-BRM) scale, showing decreased QoL (Paterson et al, 2005). In an associated study, Trask looked at 16 patients in a longitudinal analysis which showed reductions in QoL (Trask et al, 2004). Bender assessed QoL as part of a trial with 16 patients, and showed a significant reduction in physical well-being associated with high-dose interferon alpha-2b therapy using the Functional Assessment of Cancer Therapy - General (FACT-G) scale (Bender et al, 2000).

The largest available study that used a validated QoL measure is by Rataj et al (2005) that reported a study of 110 melanoma patients receiving interferon alpha- $2 \mathrm{~b}$ patients following radical

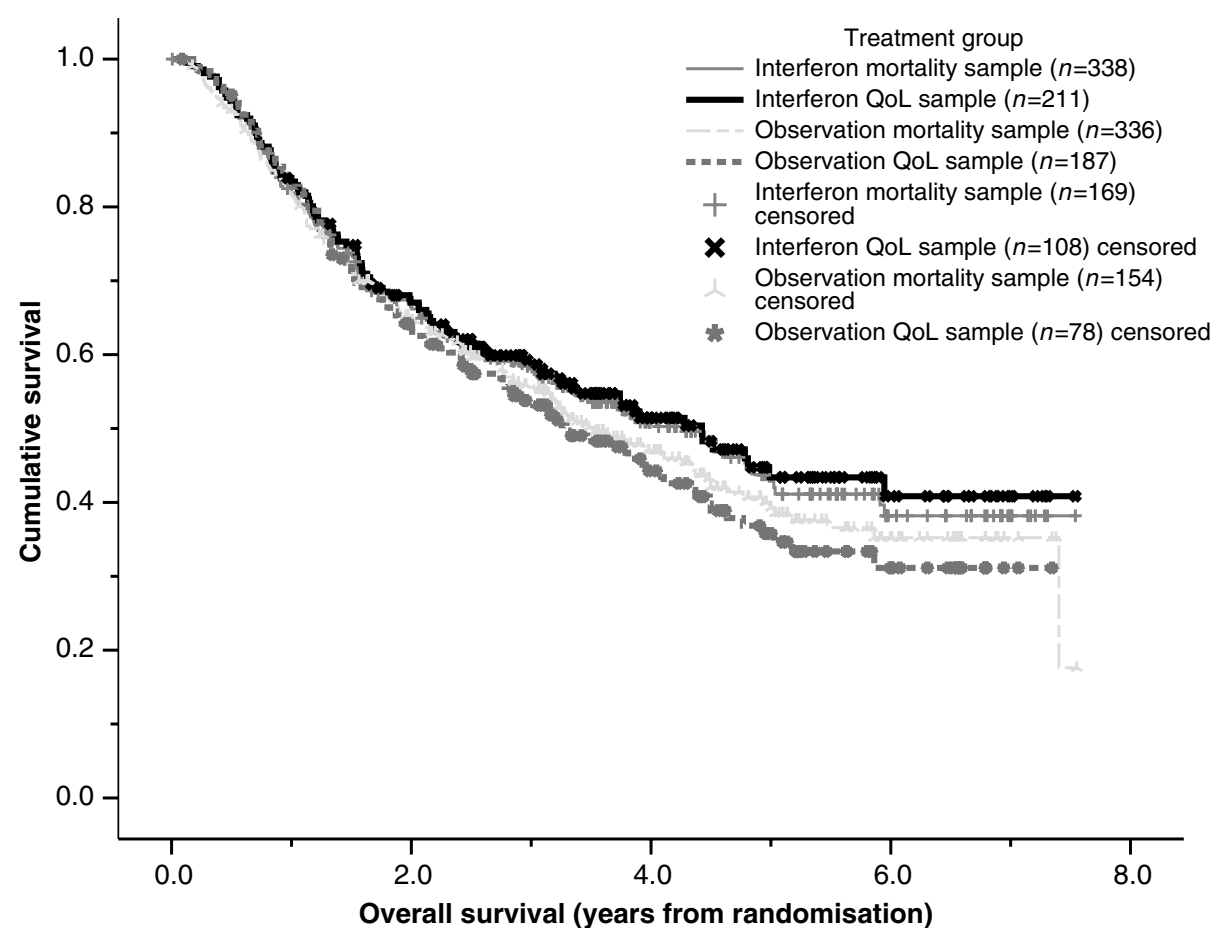

Figure 2 Kaplan-Meier plot of overall survival by treatment group HRQoL follow-up sample. 
Table 3 Baseline and follow-up EORTC-QLQ-C30 vl function scores by group $(n=398)$

\begin{tabular}{|c|c|c|c|c|c|c|c|c|}
\hline Group & \multicolumn{3}{|c|}{ Baseline } & \multicolumn{3}{|c|}{ Follow-up } & $\begin{array}{l}\text { Adjusted difference } \\
\qquad(95 \% \mathrm{Cl})\end{array}$ & $P$-value \\
\hline IFN & 211 & 88.7 & $(19.8)$ & 85.9 & $(20.2)$ & 2.2 & 2.1 & 0.144 \\
\hline OBS & 187 & 89.0 & $(19.4)$ & 88.1 & $(16.8)$ & & $(-0.7$ to 4.5$)$ & \\
\hline \multicolumn{9}{|c|}{ Role functioning } \\
\hline \multicolumn{9}{|c|}{ Emotional functioning } \\
\hline IFN & 210 & 79.0 & $(20.7)$ & 79.4 & $(19.0)$ & 4.7 & 4.5 & 0.003 \\
\hline OBS & 187 & 79.7 & $(21.1)$ & 84.1 & $(17.5)$ & & $(1.6-7.4)$ & \\
\hline \multicolumn{9}{|c|}{ Cognitive functioning } \\
\hline IFN & 211 & 89.5 & $(19.1)$ & 87.3 & $(16.6)$ & 5.1 & 4.1 & 0.001 \\
\hline \multicolumn{9}{|c|}{ Global health status/Qol } \\
\hline IFN & 210 & 71.6 & $(20.8)$ & 67.5 & $(19.2)$ & 7.2 & 5.9 & 0.001 \\
\hline OBS & 187 & 73.8 & $(19.3)$ & 74.7 & $(16.5)$ & & $(3.1-8.7)$ & \\
\hline
\end{tabular}

For the EORTC QLQ-C30 vI function scales a higher score represents a better level of functioning. The treatment group difference in mean follow-up scores is adjusted for baseline score and overall survival status (dead or censored). A positive follow-up difference implies the observation (OBS) group has a better level of functioning at follow-up, than the interferon (IFN) group.

surgery. Using the EORTC QLQ-C30 (version 2.0) they found treatment had an impact on physical function, social life, emotional functioning and cognitive functioning. Direct comparisons of that study with this, are not possible due to limited reporting in their paper.

Other work has been undertaken looking at QoL in melanoma patients receiving interferon; however, this has been undertaken with a completely different approach. Kilbridge et al (2001), for instance, used the standard gamble technique to value a series of health states describing the QOL associated with interferon toxicity, melanoma recurrence and disease-free health. Their study, based on 107 patient interviews, showed that the side effects from interferon treatment reduced QoL, from 0.96 for the disease-free health state to 0.81 from severe side effects.

The Kilbridge utility estimates have been combined with mortality data from the ECOG 1684 trial $(n=280)$ to produce a quality-adjusted survival analysis (Kilbridge et al, 2002) and a cost-utility analysis (Crott et al, 2004). Other analyses have used other utility estimates to describe treatment and post treatment QoL for interferon patients (Cole et al, 1996; Hillner et al, 1997; Lafuma et al, 2001); however, the utility figures were assigned by the researchers rather than derived from patients.

All of these utility-based studies show that a decrease in QoL during interferon treatment is more than offset by improved QoL owing to reduced recurrence and reduced mortality. Consequently, when these utility estimates are combined with the ECOG 1684 data, results tend to show that treatment with high-dose interferon is cost-effective compared to other technologies (Hillner et al, 1997; Lafuma et al, 2001; Crott et al, 2004). These results are in contrast to this study, which shows that while median survival is around 1-year longer, combining QoL with mortality proves the IFN group to be only marginally better $(0.074$ QALYs, $P=0.752)$. This produces an incremental cost-effectiveness ratio of $£ 41432$ per QALY. Using a funding threshold of $£ 30000$ per QALY which is at the higher end of a range used within the United Kingdom
(National Institute for Clinical Excellence, 2004), these results show that low-dose extended duration interferon therapy is unlikely to be considered cost-effective.

There are several reasons for these differences. Firstly, AIMHigh is a study of low dose interferon therapy, whereas ECOG 1684 is a study of high-dose therapy. Consequently, QoL, survival and recurrence might be expected to differ. Secondly, the utility figures are derived in completely different ways. Our study used a generic preference based outcome measure (EQ-5D) to gather data prospectively from within the trial, from which general population utilities values were applied from a standard algorithm. Kilbridge et al (2001) generated utility values from melanoma patients by asking them to value health states describing various treatment scenarios. Thirdly, our study estimates cost-effectiveness at 5 years, while the modelling studies look at longer time scales; 35 years in one case (Crott et al, 2004). This is an important difference as shorter time frames generate higher incremental costeffectiveness ratios. While extrapolation of our results is possible, the lower final year QALY estimate (Table 5) implies that even worse cost-effectiveness results may be produced, if such an analysis were undertaken.

We should also consider the deficiencies associated with our study. Only $66 \%$ of patients in the trial had a baseline EORTC QLQ-C30 assessment. Despite this, there appears to be no systematic difference between patients included in our QoL analysis, and those excluded. Another problem was that the number and timing of QoL assessments completed, varied. This led us to undertake a simple analysis, using a summary measure of QoL based on the average scores. As assessments were more frequent during interferon treatment in order to capture the impact of side effects, the results will be weighted toward the early months of treatment. However, repeating the analysis with average follow-up over the first 2 years as the outcome, rather than the total follow-up gave almost identical results to the longer follow-up (data not shown). 
We followed the advice of Cox et al (1992) for QoL studies which recommended simplicity of design, analysis and presentation of QoL assessments. Therefore, we decided to use a simple approach and not the simultaneous assessment of QoL and survival. There are several approaches to the simultaneous assessment of QoL and survival including: QALYs (for which we employed the EQ-5D), Q-TWiST (quality-adjusted time with spent with symptoms of disease and toxicity of treatment) and multistate survival analysis

Table 4 Baseline and mean follow-up EORTC-QLQ-C30 vI Symptom scores by group $(n=398)$

\begin{tabular}{|c|c|c|c|c|c|c|c|c|}
\hline & \multicolumn{3}{|c|}{ Baseline } & \multicolumn{2}{|c|}{ Follow-up } & \multirow{2}{*}{$\begin{array}{l}\text { Follow-up } \\
\text { Difference }\end{array}$} & \multirow{2}{*}{$\begin{array}{c}\text { Adjusted } \\
\text { difference } \\
(95 \% \mathrm{Cl})\end{array}$} & \multirow[b]{2}{*}{$P$-value } \\
\hline & $n$ & Mean & s.d. & Mean & s.d. & & & \\
\hline \multicolumn{9}{|l|}{ Fatigue } \\
\hline IFN & 211 & 21.7 & $(23.3)$ & 29.0 & $(21.7)$ & -10.8 & -9.1 & 0.001 \\
\hline OBS & 187 & 18.5 & $(21.3)$ & 18.2 & $(17.7)$ & & $(-12.1$ to 6.1$)$ & \\
\hline \multicolumn{9}{|c|}{$\begin{array}{l}\text { Nausea and } \\
\text { vomiting }\end{array}$} \\
\hline IFN & 211 & 2.4 & $(6.9)$ & 6.1 & $(10.8)$ & -3.4 & -3.4 & 0.001 \\
\hline OBS & 187 & 2.0 & (8.6) & 2.7 & $(8.6)$ & & $(-5.3$ to -1.6$)$ & \\
\hline \multicolumn{9}{|l|}{ Pain } \\
\hline IFN & 211 & 19.8 & $(25.3)$ & 16.8 & $(21.1)$ & -2.7 & -0.1 & 0.937 \\
\hline OBS & 187 & 14.0 & (20.7) & 14.0 & $(18.2)$ & & $(-3.4$ to 3.1$)$ & \\
\hline \multicolumn{9}{|c|}{ Dyspnoea } \\
\hline IFN & 211 & 5.1 & $(12.4)$ & 12.2 & $(16.2)$ & -2.7 & -3.7 & 0.010 \\
\hline OBS & 187 & 6.6 & $(15.4)$ & 9.5 & $(16.8)$ & & $(-6.5$ to -0.9$)$ & \\
\hline \multicolumn{9}{|l|}{ Insomnia } \\
\hline IFN & 211 & 21.6 & $(25.8)$ & 23.1 & $(23.6)$ & -3.8 & -3.2 & 0.123 \\
\hline OBS & 187 & 20.1 & $(29.6)$ & 19.4 & (23.4) & & $(-7.2$ to 0.9$)$ & \\
\hline \multicolumn{9}{|c|}{ Appetite loss } \\
\hline IFN & 210 & 4.6 & $(\mid 4.8)$ & 12.4 & $(20.8)$ & -5.8 & -6.2 & 0.001 \\
\hline OBS & 187 & 4.8 & $(15.3)$ & 6.6 & $(15.1)$ & & $(-9.5$ to -3.0$)$ & \\
\hline \multicolumn{9}{|c|}{ Constipation } \\
\hline IFN & 211 & 6.5 & $(17.7)$ & 7.9 & $(16.6)$ & -3.7 & -3.3 & 0.011 \\
\hline OBS & 187 & 5.2 & $(14.8)$ & 4.1 & $(10.8)$ & & $(-5.8$ to -0.7$)$ & \\
\hline \multicolumn{9}{|c|}{ Diarrhoea } \\
\hline IFN & 211 & 3.6 & $(12.7)$ & 8.8 & $(13.6)$ & -4.5 & -4.4 & 0.001 \\
\hline OBS & 187 & 3.6 & $(10.9)$ & 4.3 & $(11.8)$ & & $(-6.9$ to -2.0$)$ & \\
\hline \multicolumn{9}{|c|}{$\begin{array}{l}\text { Financial } \\
\text { difficulties }\end{array}$} \\
\hline IFN & 210 & |5.| & $(27.3)$ & 11.8 & $(23.6)$ & -4.8 & -4.7 & 0.001 \\
\hline OBS & 187 & 14.3 & (28.7) & 7.0 & $(15.6)$ & & $(-7.4$ to -2.0$)$ & \\
\hline
\end{tabular}

For the EORTC QLQ-C30 vI symptom scales a higher score represents a worse level of symptoms. The treatment group difference in mean follow-up scores is adjusted for baseline score and overall survival status (dead or censored). A negative follow-up difference implies the observation (OBS) group has a lower/better level of symptoms, at follow-up, than the interferon (IFN) group.
(Billingham et al, 1999). The latter two approaches would require the definition of a finite number of health states in terms of the 15 EORTC QLC-30 dimension scores. We felt it was very difficult to define a set of finite, mutually exclusive and exhaustive health states that are clinically meaningful and fully describe the experiences of patients with malignant melanoma using the 15 dimensions of the EORTC QLC-30.

We assumed that the missing QoL data are missing at random and that dropout was noninformative. We found that the dropout rates and survival experience were similar across the treatment arms and believe that the between-treatment comparisons of QoL remain unbiased. We also included a term for overall survival status in our regression model to adjust for whether the patient was alive or dead during follow-up. This term should take into account that patients who died during follow-up may have a different average QoL at follow-up than patients who were alive or censored.

Further loss of data was present when the economic results are considered, such that data on only 111 patients were available for analysis. Even for these patients, missing data meant that imputation was required to produce a rectangular data set. While differences between this economic subsample and the full sample are not statistically significant, we are limited in our ability to detect differences between the two arms due to the smaller sample size. This problem is perhaps compounded by the possible insensitivity of the EQ-5D seen in several studies (Harper et al, 1997; Nicholl et al, 2001; Patel et al, 2004). Taken together, the lack of a clear pattern in the QALY estimates shown in Table 5 is difficult to interpret.

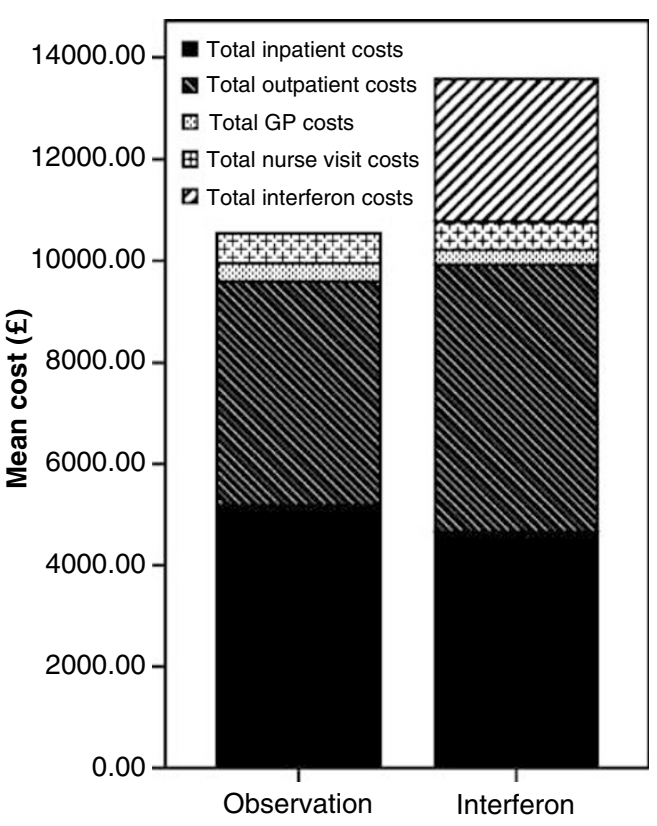

Figure 3 Total costs over 5 years within the two groups $(n=111)$.

Table 5 Profile of quality-adjusted life years for interferon and control patients

\begin{tabular}{lcccccc}
\hline & \multicolumn{5}{c}{ Mean QALYs (s.d.) } \\
\cline { 2 - 6 } & Year I & Year 2 & Year 3 & Year 4 & Year 5 & Total \\
\hline Observation group $(n=51)$ & $0.79(0.18)$ & $0.62(0.27)$ & $0.43(0.37)$ & $0.29(0.35)$ & $0.19(0.31)$ & $2.33(1.25)$ \\
Interferon group $(n=60)$ & $0.76(0.22)$ & $0.67(0.29)$ & $0.50(0.34)$ & $0.31(0.35)$ & $0.16(0.29)$ & $2.40(1.19)$ \\
\hline
\end{tabular}

Quality-adjusted life years are calculated by multiplying quality of life by length of life, such that I year in full health is equivalent to one quality-adjusted life year (QALY). When I year produces less than one QALY, this reflects less than full health, for example, 0.5 QALYs is I year in a health state valued at 0.5 , which is deemed to be equivalent to 6 months ( 0.5 years) in full health. 
Looking beyond this study, it is difficult to cast light on other QoL evidence and economic evaluations, as methods are different, as too are the interferon dosing regimens. However, given that such a clear picture of QoL is produced with the EORTC QLQ-C30 we would recommend its use for further studies of interferon treatment. The much cited ECOG 1684 study did not incorporate prospective QoL assessment, and so subsequent evaluations have had to add on supplementary studies. While several improvements to future economic evaluations have been suggested (Crott, 2004), basing future evaluations on trial-based QoL and/or utility estimates would appear to be important given the differences identified here.

Few studies have assessed the impact of interferon therapy on health related QoL using validated instruments. These results

\section{REFERENCES}

Aaronson NK, Ahmedzai S, Bergman B, Bullinger M, Cull A, Duez NJ, Filiberti A, Flechtner H, Fleishman DB, De Haes JCJM, Kaasa S, Klee M, Osoba D, Razavi D, Rofe P, Schraub S, Sneeuw K, Sullivan M, Takeda F, for the European Organization for Research and Treatment of Cancer (1993) The European Organisation for Research and Treatment of Cancer QLQ-C30: a quality-of-life instrument for use in international trials in clinical oncology. J Natl Cancer Inst 85: 365-376

Altman DG (1991) Practical Statistics for Medical Research. London: Chapman and Hall

Ascierto PA, Scala S, Ottaiano A, Simeone E, de Michele I, Palmieri G, Castello G (2006) Adjuvant treatment of malignant melanoma: Where are we? Crit Rev Oncol Hematol 57: 45-52

Bender CM, Yasko JM, Kirkwood JM, Ryan C, Dunbar-Jacob J, Zullo T (2000) Cognitive function and quality of life in interferon therapy for melanoma. Clin Nurs Res 9: $352-363$

Billingham LJ, Abrahams KR, Jones DR (1999) Methods for the analysis of quality-of-life and survival data in health technology assessment. Health Technology Assess 3(10): 1-152

British Medical Association (2002) British National Formulary Volume 44. London: British Medical Association

Cole BF, Gelber RD, Kirkwood JM, Goldhirsch A, Barylack E, Borden E (1996) Quality-of-life-adjusted survival analysis of interferon alfa-2b adjuvant treatment of high-risk resected cutaneous melanoma: an Eastern Cooperative Oncology Group Study. J Clin Oncol 14: 2666-2673

Cox DR, Fitzpatrick R, Fletcher AE, Gore M, Jones DR, Spiegelhalter DJ (1992) Quality-of-life assessment: Can we make it simple? J Roy Stat Soc, Ser A 155: 353-375

Crott R (2004) Cost-effectiveness and cost utility of adjuvant interferon á in cutaneous melanoma; a review. Pharmacoeconomics 22: 569-580

Crott R, Ali F, Burdette-Radoux S (2004) Cost-utility of adjuvant high-dose interferon alpha therapy in stage III cutaneous melanoma in Quebec. Value Health 7: 423-432

Dolan P (1997) Modeling valuations for EuroQol health states. Med Care 35: $1095-1108$

Drummond MF, O’Brien B, Stoddart GL, Torrance GW (1997) Methods for the Economic Evaluation of Health Care Programmes. Oxford: Oxford Medical Publications

Fayers P, Aaronson N, Bjordal K, Sullivan M, on behalf of the EORTC Quality of Life Study Group (1995) EORTC QLQ-C30 Scoring Manual. Belgium: European Organisation for Research and Treatment of Cancer

Hancock BW, Harris S, Wheatley K, Gore M (2000) Adjuvant interferon-alpha in malignant melanoma: current status. Cancer Treat Rev 26: $81-89$

Hancock BW, Wheatley K, Harris S, Ives N, Harrison G, Horsman JM, Middleton MR, Thatcher N, Lirgan PC, Marsden JR, Burrows L, Gore M (2004) Adjuvant interferon in high-risk melanoma: the AIM-HIGH show that interferon has significant effects on QoL and symptomatology. Our associated economic analysis also showed that overall, adjuvant low-dose extended duration interferon therapy does not appear cost-effective in this patient group in the UK context.

\section{ACKNOWLEDGEMENTS}

We gratefully acknowledge the contribution of the many investigators and patients participating in the trial. The study was supported by a Grant from Roche Products Ltd, and provided the interferon alpha-2a for the first 3 years of the study.

study - United Kingdom Coordinating Committee on Cancer Research randomized study of adjuvant low-dose extended-duration interferon alfa-2a in high-risk resected malignant melanoma. J Clin Oncol 22: 53-61

Harper R, Brazier JE, Waterhouse JC, Walters SJ, Jones NM, Howard P (1997) Comparison of outcome measures for patients with chronic obstructive pulmonary disease (COPD) in an outpatient setting. Thorax 52: $879-887$

Heyting A, Tolboom JT, Essers JG (1992) Statistical handling of drop-outs in longitudinal clinical trials. Stat Med 11: 2043-2061

Hillner BE, Kirkwood JM, Atkins MB, Johnson ER, Smith TJ (1997) Economic analysis of adjuvant interferon alfa- $2 \mathrm{~b}$ in high-risk melanoma based on projections from Eastern Cooperative Oncology Group 1684. J Clin Oncol 15: $2351-2358$

Kilbridge KL, Cole BF, Kirkwood JM, Haluska FG, Atkins MA, Ruckdeschel JC, Sock DE, Nease RF, Weeks JC (2002) Quality-of-life-adjusted survival analysis of high-dose adjuvant interferon alfa- $2 \mathrm{~b}$ for high-risk melanoma patients using intergroup clinical trial data. J Clin Oncol 20: 1311-1318

Kilbridge KL, Weeks JC, Sober AJ, Haluska FG, Slingluff CL, Atkins MB, Sock DE, Kirkwood JM, Nease RF (2001) Patient preferences for adjuvant interferon alfa-2b treatment. J Clin Oncol 19: 812-823

Lafuma A, Dreno B, Delaunay M, Emery C, Fagnani F, Hieke K, Bonerandi JJ, Grob JJ, for the French Cooperative Group on Melanoma (2001) Economic analysis of adjuvant therapy with interferon alpha-2a in stage II malignant melanoma. Eur J Cancer 37: 369-375

Matthews JNS, Altman DG, Campbell MJ, Royston PG (1990) Analysis of serial measurements in medical research. BMJ 300: 230-235

Molife R, Hancock BW (2002) Adjuvant therapy of malignant melanoma. Crit Rev Oncol Hematol 44: 81-102

National Institute for Clinical Excellence (2004) Guide to the Methods of Technology Appraisal. London: National Institute for Clinical Excellence

Netten A, Curtis L (2003) Unit Costs of Health and Social Care 2003. Canterbury: Personal and Social Services Research Unit

Nicholl CR, Lincoln NB, Francis VM, Stephan TF (2001) Assessing quality of life in people with multiple sclerosis. Disabil Rehabil 23: 597-603

Patel A, Knapp M, Evans A, Perez I, Kalra L (2004) Training care givers of stroke patients: economic evaluation. BMJ 328: 1102

Paterson AG, Trask PC, Wagner LI, Esper P, Redman B (2005) Validation of the FACT-BRM with interferon-alpha treated melanoma patients. Qual Life Res 14: 133-139

Rataj D, Krajewska-Kulak E, Jankowiak B, van Damme-Ostapowicz K, Nowecki ZI, Rutkowski P, Niczyporuk W (2005) Quality-of-life evaluation in an interferon therapy after radical surgery in cutaneous melanoma patients. Cancer Nurs 28: 172-178

Trask PC, Paterson AG, Esper P, Pau J, Redman B (2004) Longitudinal course of depression, fatigue, and quality of life in patients with high risk melanoma receiving adjuvant interferon. Psychooncology 13: 526-536 